Scientia Agricola

http://dx.doi.org/10.1590/0103-9016-2014-0225

\title{
Mixed effect models for predicting breast height diameter from stump diameter of Oriental beech in Göldağ
}

\author{
Ilker Ercanli* ${ }^{*}$ Alkan Gunlu, Emin Zeki Başkent \\ Çankııı Karatekin University/Forest Faculty, Bademlik Avenue \\ -18200 - Cankırı - Turkey. \\ *Corresponding author <ilkerercanli@karatekin.edu.tr>
}

Edited by: Thomas Kumke

Received June 29, 2014

Accepted November 20, 2014

\begin{abstract}
Diameter at breast height (DBH) is the simplest, most common and most important tree dimension in forest inventory and is closely correlated with wood volume, height and biomass. In this study, a number of linear and nonlinear models predicting diameter at breast height from stump diameter were developed and evaluated for Oriental beech (Fagus orientalis Lipsky) stands located in the forest region of Ayancık, in the northeast of Turkey. A set of 1,501 pairs of diameter at breast height-stump measurements, originating from 70 sample plots of even-aged Oriental beech stands, were used in this study. About $80 \%$ of the otal data (1,160 trees in 55 sample plots) was used to fit a number of linear and nonlinear model parameters; the remaining 341 trees in 15 sample plots were randomly reserved for model validation and calibration response. The power model data set was found to produce the most satisfactory fits with the Adjusted Coefficient of Determination, $R_{\text {adj }}^{2}$ (0.990), Root Mean Square Error, RMSE (1.25), Akaike's Information Criterion, AIC (3820.5), Schwarz's Bayesian Information Criterion, BIC (3837.2), and Absolute Bias (1.25). The nonlinear mixed-effect modeling approach for power model with $\mathrm{R}_{\mathrm{adj}}^{2}$ (0.993), AIC (3598), BIC (3610.1), Absolute Bias (0.73) and RMSE (1.04) provided much better fitting and precise predictions for DBH from stump diameter than the conventional nonlinear fixed effect model structures for this model. The calibration response including tree DBH and stump diameter measurements of the four largest trees in a calibrated sample plot in calibration produced the highest Bias, $-5.31 \%$, and RMSE, $-6.30 \%$, the greatest reduction percentage. Keywords: DBH, prediction, random parameters, calibration
\end{abstract}

\section{Introduction}

Diameter at breast height $(\mathrm{DBH})$ is the simplest, most common and most important tree dimension in forest inventory (Vanclay, 1994), since DBH is closely correlated to other dendrometric tree attributes, i. e. the wood volume, height and biomass. The diameter cannot be measured at breast height in certain forest applications, such as illegal timber cut studies or unrecorded data from harvested stands. In these forest applications, the stump diameter, which is measured at $0.3 \mathrm{~m}$, remains an indicator of tree size (Randall et. al., 1984; Corral-Rivas et. al., 2007). DBH is initially predicted from the stump diameters directly measured on the logged trees, and then the tree volume and other tree attributes can be estimated from the DBH predicted (Bylin, 1982; Wharton, 1984; Parresol, 1998).

The relationship between the $\mathrm{DBH}$ and the stump diameter measured greatly depends on stand structures, where these stand conditions can be differentiated by site quality, stand density and stand ages. Accordingly, there is a nested structure (stand and plot), which can cause a lack of independence among diameter measurements with highly correlated data obtained from different sample plots (West et. al., 1984; Gregoire, 1987).

In view of the disadvantages of the traditional modeling technique with the violation of assumptions in the regression estimation to fit the correlated hierarchical data structure (Searle et. al., 1992; Gregoire, 1987; Judge et. al., 1988), the linear and nonlinear mixed effect fitting technique have generally been accepted as the alternative modeling approach to justify these serial correlation problems among trees (Lappi and Bailey, 1988; Gregoire and Schabenberger, 1996; Hall and Clutter, 2004; Temesgen et. al., 2008). However, there are few studies that include the use of the linear and nonlinear mixed effect fitting procedure to fit the relationships between $\mathrm{DBH}$ and the stump diameter.

Despite the importance of predicting $\mathrm{DBH}$ in forestry applications, mixed effect modeling for the relationship between DBH and stump diameter in forestal ecosystems has been poorly understood. Thus, the objective of the authors in the present study is to (i) develop a number of linear and nonlinear mixed effect models for predicting DBH from stump diameter for Oriental beech (Fagus orientalis Lipsky) stands located in the forest region of Ayancik, northeastern of Turkey, and (ii) evaluate a number of calibration strategies including different numbers of subsample trees for specifying the sampling unit-specific effect incorporated into the DBH predictions.

\section{Materials and Methods}

\section{Data Description}

This study was performed on Oriental beech stands located in the Göldağ planning unit, Sinop, in the northeast of Turkey (647000-650000 E. 4629000-4632000 N. UTM ED 50 datum Zone $36 \mathrm{~N})$. The altitudes of the study area ranged from $500 \mathrm{~m}$ to $970 \mathrm{~m}$, with an average of $775 \mathrm{~m}$. The study area was characterized geomorpho- 
logically as being steep terrain land with moderate and steep slopes ranging from $10 \%$ to $60 \%$, with an average of $26 \%$.

In the stands studied, 70 sample plots were randomly selected so as to guarantee that various stand conditions including variability in site quality, age and stand density were found in the Oriental beech stands. The Oriental beech stands sampled were naturally regenerated and uniformly stocked stands $160-90 \%$ tree layer cover), without any evidence of historical damage such as fire or storm. In the Oriental beech stands studied, the Regional Forest Directory used moderate thinning regime and patch cutting procedures to guarantee the stand presence of the Oriental beech trees.

The size of circular plots ranged from 0.04 to 0.08 ha, which were dependent on the stand crown closure in order to achieve a minimum of 30-40 trees in sample plots. In each sample plot, the diameter at breast height (DBH) and stump diameter at $0.3 \mathrm{~m}$ stump height were measured to within a precision limit of $0.1 \mathrm{~cm}$ with calipers for every living tree with a DBH greater than 4 $\mathrm{cm}$. Thus, in total $1501 \mathrm{DBH} /$ stump diameter pairs were used to develop the statistical models. Of special importance was the random splitting into two data sets as follows: the model fitting data and the validation data set.

About $80 \%$ of total data $(1,160$ trees in 55 sample plots) was used to develop DBH-stump diameter models. The remaining 341 trees in 15 sample plots (20\% of total data) were randomly reserved to evaluate calibration responses of the nonlinear mixed effect model which was selected as the best predictive model. The mean, standard deviations, minimum, maximum for model fitting and validation data set are presented in Table 1 .

\section{Analyzed Models}

In this study, the allometric models used to develop statistical models for predicting $\mathrm{DBH}$ from stump diameter were both linear (simple, M1, and quadratic, M2, model) and nonlinear models (power, M3 and com- pound, M4, model). Model structures analyzed in this study are shown in Table 2.

The linear regression models, M1-M2, were fitted by ordinary least squares using PROC REG procedure and the nonlinear models, M3-M4, were fitted by the non-linear regression analysis with the Marquadt algorithm using the PROC NLIN procedure of the SAS/ETS V9 software (SAS Institute Inc, 2004).

\section{Model Selection and Evaluation}

The linear and nonlinear models predicting $\mathrm{DBH}$ from stump diameter were evaluated as to their accuracy and precision by using the following five statistical criteria: Akaike's Information Criterion (AIC), Schwarz's Bayesian Information Criterion (BIC), the Root Mean Square Error (RMSE), the Absolute Bias, and the Adjusted Coefficient of Determination $\left(\mathrm{R}_{\text {adj }}^{2}\right)$ using the following equations:

$A I C=-2 \log L+2 q$

$B I C=2 \log (L)+q \log (N)$

$R M S E=\sqrt{\frac{\sum_{i=1}^{N} \sum_{j=1}^{n_{i}}\left(d b h_{i j}-{\widehat{d b h_{i j}}}^{2}\right.}{\sum_{i=1}^{N} n_{i}-p}}$

Absolute Bias $=\frac{\sum_{i=1}^{N} \sum_{j=1}^{n_{i}}\left|d b h_{i j}-\widehat{d b h}_{i j}\right|}{\sum_{i=1}^{N} n_{i}}$

Adjusted coefficient of determination $\left(\mathrm{R}_{\mathrm{adj}}^{2}\right)$

$$
=1-\frac{\sum_{i=1}^{N} \sum_{j=1}^{n_{i}}\left(d b h_{i j}-\widehat{d b h}_{i j}\right)^{2}\left(\sum_{i=1}^{N} n_{i}-1\right)}{\sum_{i=1}^{N} \sum_{j=1}^{n_{i}}\left(d b h_{i j}-\overline{d b h}_{i}\right)^{2}\left(\sum_{i=1}^{N} n_{i}-p\right)}
$$

where: $L$ is the maximum value of the log likelihood function, $q$ is the number of variance components, $N$

Table 1 - Summary statistics for sample trees and sample plots originated from fitting, validation and all data.

\begin{tabular}{|c|c|c|c|c|c|}
\hline Variables & & Min. & Mean & Max. & Std. Deviation \\
\hline & Fitting & 4.50 & 19.44 & 68.00 & 11.96 \\
\hline \multirow[t]{3}{*}{ Diameter at Breast Height } & Validation & 5.70 & 23.27 & 61.40 & 13.27 \\
\hline & All & 4.50 & 20.31 & 68.00 & 12.37 \\
\hline & Fitting & 5.10 & 22.33 & 77.00 & 13.45 \\
\hline \multirow[t]{3}{*}{ Stump Diameter } & Validation & 6.40 & 26.59 & 68.80 & 15.13 \\
\hline & All & 5.10 & 23.29 & 77.00 & 13.96 \\
\hline & Fitting & 11.8 & 24.5 & 32.8 & 5.2 \\
\hline \multirow[t]{3}{*}{ Site Index } & Validation & 10.9 & 20.6 & 33.7 & 4.8 \\
\hline & All & 10.9 & 22.6 & 33.7 & 4.9 \\
\hline & Fitting & 32 & 65 & 124 & 15.8 \\
\hline \multirow[t]{3}{*}{ Stand Age } & Validation & 28 & 58 & 114 & 12.8 \\
\hline & All & 28 & 65 & 112 & 14.9 \\
\hline & Fitting & 0.25 & 0.44 & 1.15 & 0.10 \\
\hline \multirow[t]{2}{*}{ Density Index } & Validation & 0.20 & 0.50 & 1.22 & 0.12 \\
\hline & All & 0.20 & 0.48 & 1.22 & 0.13 \\
\hline
\end{tabular}


Table 2 - Equation structure of linear and nonlinear models analyzed in this study.

\begin{tabular}{lcc}
\hline & Model Name & Linear and Nonlinear Model Structure (Fixed effects model) \\
\hline Model 1 & Simple & Linear Models \\
Model 2 & Quadratic & $d b h=b_{0}+b_{1} d_{s d}+\varepsilon_{i j}$ \\
\hline Model 3 & Power & Nonlinear Models \\
Model 4 & Compound & $d b h=b_{0}+b_{1} d_{s d}+b_{2} d_{s d}^{2}+\varepsilon_{i j}+\varepsilon_{j}$ \\
\hline
\end{tabular}

$\mathrm{dbh}$ : diameter at breast height outside bark (1.3 $\mathrm{m}$ above ground, $\mathrm{cm})$; dsd: stump diameter $(0.3 \mathrm{~m}$ above ground $\mathrm{cm})$.

is the number of sample plots, $n_{i}$ is the number of trees in the ith plot, $d b h_{i j}$ and $d \widehat{d b h}_{i j}$ are the $j$ th observed and estimated DBH in ith sample plot, respectively, and $\overline{d b h}_{i}$ is the mean DBH in the ith sample plot. Smaller values of $A I C, B I C, R M S E$ and the Absolute Bias indicate better model fit results. Higher values of $\mathrm{R}_{\text {adj }}^{2}$ (the adjusted coefficient of determination) with 1.0 as the ideal value give the predictable proportion of the variance of the dependent variable, tree diameter at breast height, and from the independent variable, a stump diameter.

\section{The linear and nonlinear mixed effect modeling}

The linear and nonlinear fixed effect models structures (Table 2) are based on the assumption that $\varepsilon_{i j}$ is distributed with variance $\sigma^{2}$ as $\varepsilon \sim N\left(0, \sigma^{2}\right)$ In ordinary least squares and nonlinear least squares regression techniques, it is assumed that the parameters, $b_{0}, b_{1}$ and $b_{2}$, are fixed and stable across sampling units (i.e. site, stand and plots) within all populations. These models, including only fixed effects, provide the predictions of $\mathrm{DBH}$ for the mean population, which is common to all populations. However, the relationship between the $\mathrm{DBH}$ and the stump diameter varies from one stand to the next, since the stand conditions and structures are plot-specific, i. e. in terms of stand density, site and soil attributes, with significant variability.

The hierarchical sample structure resulted in the highly correlated observations for $\mathrm{DBH}$ and stump diameter data, which were measured in the same sampled plots. To fix this problem, the prediction systems based on the mixed effect modeling procedure including simultaneously both fixed and random parameters in model structure, were used to fit the DBH - stump diameter model selected as the best predictive model.

In this study, the predictions for variance components and fixed parameters of the model selected as best predictive were obtained by PROC NLMIXED and PROC MIXED procedures of the SAS/ETS V9 software (SAS Institute Inc, 2004). In nonlinear mixed effect regression, SAS PROC NLMIXED uses the maximum likelihood estimation (ML) procedure. The adaptive Gaussian quadrature was used to solve numerically the integral over the random effects in SAS NLMIXED pro- cedure, of which the detailed information was explained by Pinheiro and Bates (2000).

\section{Calibration Response Evaluation}

To calibrate the nonlinear mixed DBH models for specific sample plots, random parameters, $u i$ and $v_{i^{\prime}}$ for this plot were predicted by the best linear unbiased predictors, BLUPs (Vonesh and Chinchilli, 1997);

$\hat{b}_{i} \approx \hat{D} \hat{Z}_{i}^{T}\left(\hat{R}_{i}+Z_{i} \hat{D} \hat{Z}_{i}^{T}\right)^{-1} \hat{e}_{i j}$

where: $\hat{b}_{i}$ represents the estimated random parameters for the localized plot, $\hat{D}$ is the $2 \mathrm{x} 2$ variance-covariance matrix for the among-plot variability (common for all plots), $\hat{R}_{i}$ is the estimated kxk variance-covariance matrix for the within-plot variability, $\hat{Z}_{i}$ is the kxq matrix of partial derivatives of the function with respect to random parameters $\hat{b}_{i}, \hat{e}_{i j}$ is the residual value, which was calculated as the difference between the observed DBH value and the one predicted by using the DBH model including merely the fixed parameters in subsamples (Gregoire, 1987; Yang et. al., 2009). In particular, the comprehensive formula and explanations for the BLUP equation can be obtained from the studies of Calama and Montero (2004) and Castedo Dorado et. al. (2006).

When the calibration procedure for the mixed effect modeling approach was applied to predictions, the calibration responses of the DBH model were evaluated by using a number of sampling scenarios, which were based on subsample tree selections from the validation data set, 341 trees for Oriental beech in 15 sample plots - about $20 \%$ of the total data.

The estimation of random parameters, $\hat{b}_{i}$, was arrived at by using different sampling scenarios to obtain the DBH predictions. These sampling alternatives for calibration responses included the selection of previous trees based on certain stand size categories, i. e. largest, smallest and medium trees in the sample plots (Calama and Montero, 2004; Yang et. al., 2009). The sampling scenarios evaluated were as follows:

1) $\mathrm{DBH}$ and stump diameter of 3-5 randomly selected trees in the plot. 
2) $\mathrm{DBH}$ and stump diameter of 3-5 largest trees in the plot.

3) $\mathrm{DBH}$ and stump diameter of 3-5 medium-size trees, considered as closest to the quadratic mean diameter at breast height in the plot.

4) $\mathrm{DBH}$ and stump diameter of 3-5 smallest trees in the plot.

As calibration responses, these sampling scenarios, including the selection of the previously subsampled trees, were evaluated by a number of statistical criteria and previously defined statistics: ABSOLUTE BIAS and RMSE.

\section{Results}

Model selection and nonlinear mixed effect modeling

The parameter estimates with standard error, $t$ value and probability levels for the linear and nonlinear models that included only the fixed parameters in the model structure are presented in Table 3. All the parameters of estimates for these models were significant $(p<$ 0.05). Additionally, Table 3 shows the values of goodnessof-fit statistics, i. e. AIC, BIC, RMSE, Absolute Bias and $\mathrm{R}_{\mathrm{adj},}^{2}$ for these linear and nonlinear models studied. These goodness-of-fit statistics proposed that the M3 model, the power model, has better predictive ability in terms of $\mathrm{R}_{\text {adj }}^{2}$ (0.990), RMSE (1.25 cm), AIC (3820.5), BIC (3837.2), Absolute bias (1.25) than other models. Therefore, the base model for M3 was chosen as the best DBH-stump diameter relationship model and used for the nonlinear mixed effect modeling analysis that simultaneously included both fixed and random parameters in the model structure.

When the mixed effect modeling approach is adopted, the important issue is to determine if the parameter is to be fixed or random in the model structure. In this evaluation, the AIC and BIC statistics of the M3, including any random parameter alternatives, i.e. none whatsoever in this case, (all parameters being fixed-effect parameters), one ( $b_{0}$ or $b_{1}$ random parameter) and two random parameters (both $b_{0}$ and $b_{1}$ random parameters), are presented in Table 4 . These alternatives result in, the greatest reductions in AIC and BIC values, namely, $6.17 \%$ and $5.92 \%$; and the best predictive results were arrived at by the nonlinear mixed effect model structure when two random parameters, $b_{0}$ and $b_{1}$ were included. Thus, the general expression of the nonlinear mixed effect M3 including $b_{0}$ and $b_{1}$ as random parameters is the following:

$d b h_{i j}=\left(b_{0}+u_{i}\right) d s d_{i j}^{\left(b_{1}+v_{i}\right)}+\varepsilon_{i j}$

with $\left[\begin{array}{c}u_{i} \\ v_{i}\end{array}\right] \sim N(0, D) e_{i j} \sim N\left[0, R_{i}\right]$

where: $b_{0}$ and $b_{1}$ are fixed parameters, common to each sample plots, and $u_{i}$ and vi are the random parameters, specific to sample plots.

The parameter estimates and variance component estimates using the goodness-of-fit statistics, $\mathrm{R}_{\text {adj' }}^{2}$ AIC, BIC, Absolute bias and RMSE, in the nonlinear mixed effect M3 model are presented in Table 5. The parameters of estimates with variance components of this M3 nonlinear mixed effect model were significant at a probability level of 0.05 ( $p<0.05)$.

Figure 1 shows the plot of the standardized residuals versus the predicted DBH from the M3 nonlinear mixed effect model. The residuals of this M3 model indicate the random outlines of a residual around zero and no obvious significant trend. There is no serious breakdown of homoscedasticity that is the assumption of constant variance. Therefore, any weighting factor including alternative transformations for the DBH model developed is not required to remove the heteroscedastic variance error; since the nonlinear mixed effect M3 model provided the assumption of homoscedasticity with homogeneous prediction variances. Similarly, the results of the F-test (Figure 2) with the confidence intervals for the model's parameters showed that there was no reason to reject the null-hypothesis of intercept $=0$ and slope $=1$, which means that there were no systematic over nor underestimates in the DBH nonlinear mixed effect M3 model $(\mathrm{F}$ value $=104352.3, p$ $<0.01)$. These desirable characteristic of the residual pattern underlined the statistical acceptability for these models as statistical regression models with no bias prediction results.

Table 3 - Parameter estimations with their standard errors and probability levels for linear and nonlinear models studied.

\begin{tabular}{|c|c|c|c|c|c|c|c|c|c|c|}
\hline Model & & Estimate & Std. Error & $\mathrm{t}$ value & $p>|\mathrm{t}|$ & $R_{\mathrm{adj}}^{2}$ & AlC & $\mathrm{BIC}$ & $\begin{array}{c}\text { Absolute } \\
\text { Bias }\end{array}$ & $\begin{array}{c}\text { Root Mean Square } \\
\text { Error RMSE }\end{array}$ \\
\hline Model 1 & $\begin{array}{l}\mathrm{b}_{0} \\
\mathrm{~b}_{1}\end{array}$ & $\begin{array}{r}-0.3021 \\
0.8842\end{array}$ & $\begin{array}{l}0.0712 \\
0.0027\end{array}$ & $\begin{array}{r}-4.24 \\
323.54\end{array}$ & $\begin{array}{l}<0.0001 \\
<0.0001\end{array}$ & 0.989 & 3828.8 & 3848.1 & 0.88 & 1.25 \\
\hline Model 2 & $\begin{array}{l}\mathrm{b}_{0} \\
\mathrm{~b}_{1} \\
\mathrm{~b}_{2}\end{array}$ & $\begin{array}{l}-0.3647 \\
0.890 \\
-0.0001 \\
\end{array}$ & $\begin{array}{l}0.1284 \\
0.0102 \\
0.00017 \\
\end{array}$ & $\begin{array}{r}-2.84 \\
86.90 \\
-0.59 \\
\end{array}$ & $\begin{array}{l}<0.005 \\
<0.0001 \\
<0.005\end{array}$ & 0.989 & 3822.1 & 3840.7 & 0.89 & 1.26 \\
\hline Model 3 & $\begin{array}{l}\mathrm{b}_{0} \\
\mathrm{~b}_{1}\end{array}$ & $\begin{array}{l}0.8345 \\
1.013 \\
\end{array}$ & $\begin{array}{l}0.01028 \\
0.00344 \\
\end{array}$ & $\begin{array}{c}81.2 \\
294.41 \\
\end{array}$ & $\begin{array}{l}<0.0001 \\
<0.0001\end{array}$ & 0.990 & 3820.5 & 3837.2 & 0.88 & 1.25 \\
\hline Model 4 & $\begin{array}{l}\mathrm{b}_{0} \\
\mathrm{~b}_{1}\end{array}$ & $\begin{array}{r}4.222 \\
-25.616 \\
\end{array}$ & $\begin{array}{l}0.01071 \\
0.3085\end{array}$ & $\begin{array}{r}394.34 \\
-83.05 \\
\end{array}$ & $\begin{array}{l}<0.0001 \\
<0.0001\end{array}$ & 0.831 & 5952.5 & 5967.7 & 2.97 & 4.92 \\
\hline
\end{tabular}


Table 4 - AIC and BIC values for M3 model structures including different random parameter alternatives for mixed modeling approach.

\begin{tabular}{lcccc}
\hline Random Parameters & $\begin{array}{c}\text { Akaike's Information } \\
\text { Criterion } \\
\text { (smaller is better) }\end{array}$ & $\begin{array}{c}\text { Akaike's Information } \\
\text { Criterion AlC } \\
\text { Reduction \% }\end{array}$ & $\begin{array}{c}\text { Schwarz's Bayesian Information } \\
\text { Criterion } \\
\text { (smaller is better) }\end{array}$ & $\begin{array}{c}\text { Schwarz's Bayesian } \\
\text { Information Criterion } \\
\text { Reduction \% }\end{array}$ \\
\hline None (all parameters as fixed effects) & 3820 & - & 3837.2 & - \\
\hline$u$ & 3700 & 3.14 & 3742 & \\
\hline$v$ and $v$ & 3650 & 4.45 & 3688 & 3.48 \\
\hline
\end{tabular}

Table 5 - Fixed parameter and variance component prediction with their standard errors and probability levels for M3 nonlinear mixed effect model.

\begin{tabular}{|c|c|c|c|c|}
\hline & Estimate & Std. Error & t value & $p>|t|$ \\
\hline \multicolumn{5}{|l|}{ Fixed parameters } \\
\hline$b_{0}$ & 0.8485 & 0.01343 & 63.18 & $<0.0001$ \\
\hline $\mathrm{b}_{1}$ & 1.0089 & 0.00453 & 222.7 & $<0.0001$ \\
\hline \multicolumn{5}{|l|}{ variance components } \\
\hline$\sigma_{u}^{2}$ & 0.00461 & 0.000134 & 34.51 & $<0.0001$ \\
\hline$\sigma_{y}^{u}$ & 0.00051 & 0.0000057 & 89.20 & $<0.0001$ \\
\hline$\sigma^{v}$ & -0.00144 & 0.000017 & -82.6 & $<0.0001$ \\
\hline$\sigma^{2}$ & 1.1372 & 0.04921 & 23.11 & $<0.0001$ \\
\hline \multicolumn{5}{|c|}{ Goodness-of-fit statistics } \\
\hline$\overline{\mathrm{R}_{\mathrm{adj}}^{2}}$ & 0.993 & Absolute Bias & 0.73 & \\
\hline Akaike's Information Criterion & 3598.0 & Root Mean Square Error & 1.04 & \\
\hline Schwarz's Bayesian Information Criterion & 3610.1 & & & \\
\hline
\end{tabular}

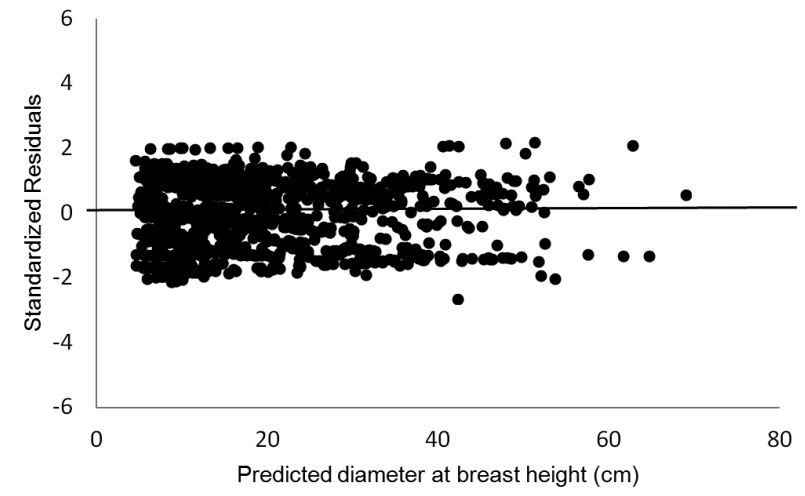

Figure 1 - Plot of studentized residuals against predicted values of diameter at breast height for Model 3.

\section{Calibration Response Evaluation}

Table 6 presents the absolute bias, RMSE and their $\%$ reduction values in these values as compared with predictions including only the fixed effect parameters of the M3 model for different sub-sample alternatives. The alternative based on the fixed-effect model structure without any random parameters resulted in the highest values of Absolute Bias and RMSE (0.95 and 1.30). However, the best predictive results with the lowest Absolute Bias and RMSE, $0.89922 \mathrm{~cm}$ and $1.22123 \mathrm{~cm}$ respectively, with the highest reduction percentage, $-5.31 \%$ and $6.30 \%$, were achieved by the sampling alternative based on the selection of the four largest trees in the calibrated sample plot.

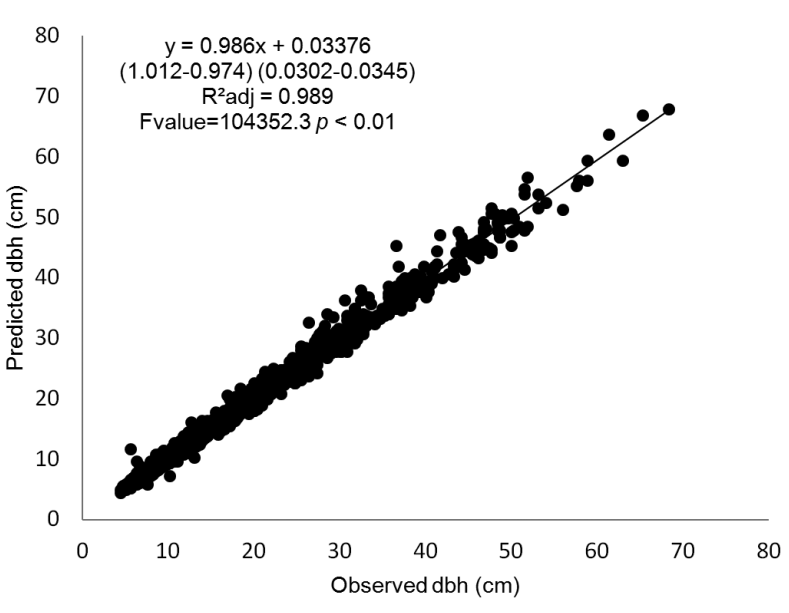

Figure 2 - Predicted dbh values against observed dbh for Model 3 with the confidence intervals for parameter shown in parenthesis.

The second-best predictive performance with $5.22 \%$ and $-5.77 \%$ reduction values for Absolute Bias and RMSE were obtained with the sampling alternative based on the three largest subsampled trees in the plots. The sampling alternatives based on the selection of randomly selected sub-sample trees produced -1.29 $\%$ to $-2.70 \%$ reduction values in absolute bias and -2.72 $\%$ to $-5.22 \%$ reduction values in RMSE as moderately predictive results. The predictive ability of sampling alternatives based on the selection of the medium and the smallest subsample trees produced the worst predictive 
Table 6 - Comparisons of nonlinear mixed effect on M3 model's predictive performance for different sub-sample size alternatives in calibration response.

\begin{tabular}{llccc}
\hline The sampling Alternatives & $\begin{array}{c}\text { Absolute } \\
\text { Bias }\end{array}$ & $\begin{array}{c}\text { \% Abs. Bias } \\
\text { Reduction }\end{array}$ & $\begin{array}{c}\text { Root Mean Square } \\
\text { Error }\end{array}$ & $\begin{array}{c}\text { \% Root Mean Square } \\
\text { Error Reduction }\end{array}$ \\
\hline Fixed Effect Model & 0.94961 & - & 1.30338 & - \\
\hline Mixed Effect Model with no calibration & 0.94327 & -0.67 & 1.28594 & -1.34 \\
\hline Mixed Effect Model with calibration based on three randomly selected trees in plot & 0.93732 & -1.29 & 1.26412 & -3.01 \\
\hline Mixed Effect Model with calibration based on four randomly selected trees in plot & 0.92395 & -2.70 & 1.26788 & -2.72 \\
\hline Mixed Effect Model with calibration based on five randomly selected trees in plot & 0.92883 & -2.19 & 1.23529 & -5.22 \\
\hline Mixed Effect Model with calibration based on three largest tree in sample plot & 0.90037 & -5.19 & 1.22812 & -5.77 \\
\hline Mixed Effect Model with calibration based on four largest trees in sample plot & 0.89922 & -5.31 & 1.22123 & -6.30 \\
\hline Mixed Effect Model with calibration based on five largest trees in sample plot & 0.90004 & -5.22 & 1.28272 & -1.58 \\
\hline Mixed Effect Model with calibration based on three medium-size trees in sample plot & 0.94181 & -0.82 & 1.29314 & -0.79 \\
\hline Mixed Effect Model with calibration based on four medium-size trees in sample plot & 0.94638 & -0.34 & 1.28908 & -1.10 \\
\hline Mixed Effect Model with calibration based on five medium-size trees in sample plot & 0.94450 & -0.54 & 1.28272 & -1.58 \\
\hline Mixed Effect Model with calibration based on three smallest trees in sample plot & 0.94181 & -0.82 & 1.29314 & -0.79 \\
\hline Mixed Effect Model with calibration based on four smallest trees in sample plot & 0.94638 & -0.34 & 1.28908 & -1.10 \\
\hline Mixed Effect Model with calibration based on five smallest trees in sample plot & 0.94450 & -0.54 & 1.28594 & -1.34 \\
\hline
\end{tabular}

results, which ranged from $-0.34 \%$ to $-0.82 \%$ for Absolute Bias and from - $0.79 \%$ to $-1.58 \%$ for RMSE (Table 6).

\section{Discussion}

In this study, certain linear and nonlinear regression models, M1-M4, were developed to predict Diameter at Breast Height from the stump diameter. Among these four models, the nonlinear power model that accounted for $99 \%$ of the total variance in DBH-stump diameter relationships in the stands studied showed the best predictive ability based on the statistics fit. In addition, the nonlinear mixed effect modeling procedure was used and the power model was fitted by including simultaneously both fixed and random effects on the DBH model structure.

The best predictive and fitting performance with the nonlinear mixed effect model structure of power model (M3) could be obtained by using the inclusion of random effects to account for the hierarchical and nested variance structure as distinct from other traditional regression fitting techniques. The nonlinear mixed effect model exhibited the homogeneous error variance for $\mathrm{DBH}$ predictions. It may be the inclusion of random parameters in models using the nonlinear mixed effect and data structure which could be obtained from the stands studied. However, Westfall (2010) stated the heteroscedastic errors for the DBHstump diameter relations, used different model structure and data origin to relate DBH-stump diameter.

We used the nonlinear mixed effect modeling procedure with the adaptive Gaussian quadrature to estimate parameters of nonlinear mixed models. Yang and Huang (2011) compared linearization through a firstorder Taylor series expansion around random effects using the first-order (FO) approximation of Sheiner and Beal (1980) and the first-order conditional expectation (FOCE) approximation of Lindstrom and Bates (1990) to estimate dominant height-age relationships. These prediction techniques, $\mathrm{FO}$ and $\mathrm{FOCE}$, for the parameters of the model may be a better choice especially, since it may be that more advanced and complicated modeling technique accounting for $\mathrm{DBH}$ variations may be required for effective and more predictive $\mathrm{DBH}$ estimations.

The calibration response based on the selection of the four largest trees in the sample plots resulted in less biased predictions, the highest reduction percentage for bias and RMSE, about 5-6 \%. The second best predictive performance for calibration response was obtained from the sampling scheme with the largest three subsample trees in plots. When the nonlinear mixed M2 model was calibrated in un-sampled stands located in different forest areas, the use of DBH and stump diameter of subsampled trees selected from the largest three or four trees in plots differed from other sampling alternatives for selecting subsampling trees.

Some attributes that have a fixed effect on sample plots did not give much more additional information and could not achieve successful predictive performance results in calibration response (Castedo-Dorado et al., 2006). In the present study, the DBH and stump diameter of medium-size and the smallest subsampled trees showed the fixed effect between plots in forest areas and species studied. Thus, the prediction results for these tree characteristics were found to be much worse than the ones achieved by other tree attributes, e.g. DBH and stump diameter of the largest sub-sample trees having a mixed effect on the DBH-stump modeling.

The similar calibration response results for the nonlinear mixed effect models were obtained by a number of studies, e.g. Krumland and Wensel (1988), and Calama and Montero (2004). However other studies, e.g. Castedo-Dorado et. al. (2006), Crecente-Campo et al. (2010) and Paulo et. al. (2011), found that the best predictive consequences for calibration response were acquired by selecting the small- 
est trees in the sample plots. The calibration responses for any mixed-effect model depend on the model structures and the characteristics of species growing in different regional and under local forest conditions. Thus, some sampling scenarios with different subsample selections, e.g. the largest or the smallest tree, can present more additive information for calibration than other sampling alternatives.

The decisive issues and crucial evaluation process for mixed-effect models are to judge some sampling alternatives, including the different selections of subsample trees, so as to obtain the best predictive performance with these models. In this evaluation of the mixed-effect model, the most important issue is to decide on was the number of trees, e.g. three or four trees or other tree quantities, that should be chosen for subsampling.

\section{Conclusions}

The model developed for the DBH and stump diameter relationships are limited to different applications for stump height, because stump diameters were measured at a stump height of $0.3 \mathrm{~m}$. The nonlinear mixed effect modeling procedure improved predictive results in DBH-stump diameter relationships as compared with fixed effect models. Also, the local calibration including the prediction of random parameters that used the subsampled tree measurements obtained from any sample plots, can be recommended for obtaining more effective and predictive results.

\section{Acknowledgment}

This work was supported by the Karadeniz Technical University Project Department, Project No: 2005.113.001.3.

\section{References}

Bylin, C.V. 1982. Estimating Dbh from Stump Diameter for 15 southern Species. USDA-Forest Service. Washington, DC, USA. (Research Note 50-286).

Calama, R.; Montero, G. 2004. Interregional nonlinear heightdiameter model with random coefficients for stone pine in Spain. Canadian Journal of Forest Research 34: 150-163.

Castedo-Dorado, F; Diéguez-Aranda, U.; Barrio, M.; Sánchez, M.; Gadow, K. von. 2006. A generalized height-diameter model including random components for radiata pine plantations in northeastern Spain. Forest Ecology and Management 229: 202- 213.

Crecente-Campo, F.; Tomé, M.; Soares, P.; Diéguez-Aranda, U. 2010. A generalized nonlinear mixed-effects height-diameter model for Eucalyptus globulus L. in northwestern Spain. Forest Ecology and Management 259: 943-952.

Corral-Rivas, J.J.; Barrio-Anta, M.; Agirre-Calderón, A.A.; Diéguez-Arandam, U. 2007. Use of stump diameter to estimate diameter at breast height and tree volume for major pine species in El Salto Durango (Mexico). Forestry 80: 29-40.

Gregoire, T.G. 1987. Generalized error structure for forestry yield models. Forest Science 33: 423-444.
Gregoire, T.G.; Schabenberger, O. 1996. A non-linear mixedeffects model to predict cumulative bole volume of standing trees. Journal of Applied Statistics 23: 257-271.

Hall, D.B.; Clutter, M. 2004. Multivariate multilevel nonlinear mixed effects models for timber yield predictions. Biometrics 60: 16-24.

Judge, G.G.; Hill, R.C.; Griffiths, W.E.; Lütkepoho, H.; Lee, T.C. 1988. Introduction to the Theory and Practice of Econometrics. John Wiley, New York, NY, USA.

Krumland, B.E.; Wensel, L.C. 1988. A generalized height-diameter equation for coastal California species. Western Journal of Applied Forestry 3: 113-115.

Lappi, J.; Bailey, R.L. 1988. A height prediction model with random stand and tree parameters: an alternative to traditional site index methods. Forest Science 34: 907-927.

Lindstrom, M.J.; Bates, D.M. 1990. Nonlinear mixed effects models for repeated measures data. Biometrics 46: 673-687.

Parresol, B.R. 1998. Prediction and error of baldcypress stem volume from stump diameter. Southern Journal of Applied Forestry 22: 69-73.

Paulo, J.A; Tomé, J.; Tomé, M. 2011. Nonlinear fixed and random generalized height-diameter models for Portuguese cork oak stands. Annals of Forest Science 68: 295-309.

Pinheiro, J.C.; Bates, D.M. 2000. Mixed Effects Models in S and S-Plus. Springer, New York, NY, USA.

Randall, B.H.; Golitz, M.; Dale, M.E. 1984. Predicting board-foot tree volume from stump diameter for eight hardwood species in Ohio. Ohio Journal of Science 84: 259-263.

Searle, S.R.; Casella, G.; McCulloch, C.E. 1992. Variance components. John Wiley, New York, NY, USA.

Sheiner, L.; Beal, S.L. 1980. Evaluation of methods for estimating population pharmacokinetic parameters. I. MichaelisMentem model: routine clinical pharmacokinetic data. Journal of Pharmacokinetics and Biopharmaceutics 8: 553-571.

Temesgen, H.; Monleon, V.J.; Hann, D.W. 2008. Analysis and comparison of nonlinear tree height prediction strategies for Douglas-fir forests. Canadian Journal of Forest Research 38: 553-565.

Vanclay, J.K. 1994. Modeling Forest Growth: Applications to Mixed Tropical Forests. CAB International, Wallingford, UK.

Vonesh, E.F.; Chinchilli, V.M. 1997. Linear and Nonlinear Models of the Analysis of Repeated Measurements. Marcel Dekker, New York, NY, USA.

West, P.W.; Ratkowsky, D.A.; Davis, A.W. 1984. Problems of hypothesis testing of regressions with multiple measurements from individual sampling units. Forest Ecology and Management 7: 207-224.

Westfall, J.A. 2010. New models for predicting diameter at breast height from stump dimensions. Northern Journal of Applied Forestry 27: 21-27.

Wharton, E.H. 1984. Predicting Diameter at Breast Height from Stump Diameters for Northeastern Tree Species. USDA-Forest Service, Washington, DC, USA. (Note NE-322).

Yang Y.; Huang, S. 2011. Comparison of different methods for fitting nonlinear mixed forest models and for making predictions. Canadian Journal of Forest Research 34 :1671-1686

Yang, Y.; Huang, S.; Trincado, G.; Meng, S.X. 2009. Nonlinear mixed effects modeling of variable-exponent taper equations for lodgepole pine in Alberta Canada. European Journal of Forest Research 128: 415-429. 